Mitteilungen aus der pharmazeutischen Abteilung des chemischen Instituts der Akademie Múnster i. W.

\title{
Ueber die Entstehung von Jodsäure und Jodaten.
}

\author{
(Beitrag zur Kenntnis der Ferricyansalze 2. Abhandlung) \\ von Georg Kassner.
}

(Eingegangen den 1. III. 1898.)

Für die Bildung und Darstellung von Jodsaure giebt es bekanntlich zahlreiche Methoden ${ }^{1}$ ). Von diesen seien als die wichtigsten genannt: 1) Behandlung von Jod mit rauchender Salpetersäure, welche indess möglichst frei von Stickstoffdioxyd sein muss. 2) Zersetzung eines jodsauren Salzes, als welches am besten Baryumjodat gewählt wird, mit Schwefelsăure. 3) Behandlung von wässriger Bromsăure oder Chlorsäure mit Jod in der Wärme.

Die einfachste und wohl auch billigste aller Methoden, ist jedenfalls die der Oxydation des Jods mit Salpetersäure. Doch scheint es unbedingt erforderlich zu sein, bei ihrer Anwendung gewisse Bedingungen streng inne $\mathrm{zu}$ halten, wenn man ohne grosse Verluste an Jod und in kürzerer Zeit ein hinreichend reines Präparat erhalten will.

So empfiehlt Connell ${ }^{2}$ ) bereits die Anwendung eines möglichst gerkumigen Kolbens, um das aufsublimierte Jod immer wieder leicht herabspülen zu können. Auch giebt derselbe an, dass die Salpetersäure möglichst konzentriert und frei von Untersalpetersauure sein solle. Dasselbe sagt $\mathrm{Duflos}{ }^{2}$ ), welcher für die Salpetersäure ein spezifisches Gewicht von 1,55 vorschreibt.

Bourson ${ }^{8}$ ) hat gefunden, dass man die Jodsăure am leichtesten erhält, wenn man 1 Teil Jod mit 4 Teilen möglichst starker Salpetersäure gelinde erwärmt, wobei die Farbe des Jods plötzlich verschwindet und nicht so viel davon verdampft, als es der Fall ist, wenn es mit einem Gemisch von Salpetersäure und salpetriger Säure behandelt wird.

Jedenfalls ist die Gegenwart von niederen Stickstoff - Sauerstoffverbindungen störend. Dasselbe kann ich von den in der pharmabis 295 .

1) Vgl. z. B. Gmelin-Kraut, Handbuch, 6. Auflage 1872, Bd. I 2, S. 292

2) Durch Gmelin-Kraut. S. 293.

8) Journal für praktische Chemie (1842), Bd. 25, S. 398 und Compt. rend. T. XIII p. 1111. 
zeutischen Abteilung des chemischen Instituts zu Münster ausgeführten präparativen Arbeiten her bestätigen. Wir machten oft die Erfahrung, dass die Herstellung der Jodsäure längere Zeit in Anspruch nalım, was offenbar nur die Folge der Anwesenheit niedriger Sauerstoffrerbindungen des Stickstoffs war.

Daher ergaben auch mehrere von mir ausgeführte Versuche, das Jod verlustlos in Jodsäure überzuführen, indem man es mit der starken Salpetersäure in Glasröhren einschmilzt und erwärmt, wobei man es also einem höheren Druck aussetzt, gar kein Resultat, trotzdem die Erhitzung auf $150^{\circ} \mathrm{C}$, , schliesslich auf $200^{\circ} \mathrm{C}$. und darüber gesteigert worden war. Nach der Abkühlung der Rohre zeigte sich in denselben kein nennenswerter Gasdruck, und das Jod war zu einem die Forın des berührten Rohrinnern zeigenden Stäbchen zusammengeschmolzen.

Wahrscheinlich wirkt das gelöste Stickstofftrioxyd reduzierend auf Jodsäure ein, sodass man in geschlossenem Gefäss aus Salpetersäure und Jod gar keine oder nur geringe Mengen der Verbindung erhält, während beim Erwärmen der letztgenannten Körper in einem mit der Luft in Berührung stehenden Kolben ein Entweichen der schädlichen Stickoxyde stattfinden kann, wodurch sich dann nach und nach die Bildung der Jodsäure vollzieht.

Uebrigens hat auch schon Kämmerer ${ }^{1}$ ) die Thatsache der Reduzierbarkeit der Todsäure durch Untersalpetersäure erwähnt. Derselbe laält die Oxydation des Jods mit Salpetersäure nur für Darstellung von Jodsüure in kleinen Mengen unter Verarbeitung von etwa 5-10 g Jod für praktisch. Will man grössere Mengen nach dieser Methode

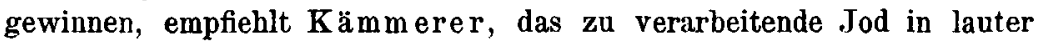
Portionen von etwa $10 \mathrm{~g}$ zu teilen, und jede für sich mit Salpetersäure zu oxydieren, weil sonst der Oxydationsprozess ein sehr langwieriger und unangenehmer wird, was ich durchaus bestätigen kann.

Im übrigen empfiehlt ${ }^{2}$ ) er behuts Gewinnung grösserer Mengen Jodsäure die Zersetzung von jodsaurem Baryum mittelst Schwefelsäure, wenn auch freilich das erhaltene Produkt noch von anhaftender Schwefelsäure befreit werden muss. Das jodsaure Baryum wieder wird nun am besten durch Einleiten von Chlor in Jodbaryumlüsung oder durch Eintragen von Jodbaryum in schmelzendes chlorsaures Kalium hergestellt.

Unter den anderen Oxydationsmitteln, welche zur Bildung von Jodsäure führen, sei noch das übermangansaure Kalium hervolgehoben,

1) Journal für praktische Chemie. Bd. 79, S. 94, 1860.

2) 1. c. S. 95 und 96. 
dessen wässrige Lösung Jodmetalle zu jodsauren Salzen zu oxydieren vermag, wie verschiedene Forscher fanden. Es schien mir nun von Interesse, $\mathrm{zu}$ untersuchen, $\mathrm{ob}$ nicht auch das für andere Oxydationszwecke vielbenutzte Ferricyankalium jodsaure Salze zu bilden vermag, wobei es mir weniger darauf ankam, eine praktisch ergiebige und vorteilhafte Methode zur Gewinnung der Jodsäure aufzufinden, als in Fortsetzung meiner früheren Mitteilungen die eigentümliche Oxydationskraft des roten Blutlaugensalzes zu beleuchten. Das Resultat der in grosser Zahl und mannigfacher Abänderung unternommenen Versuche ist die Thatsache, dass unter gewissen Bedingungen sich mit Hilfe dieses Salzes ebenfalls jodsaure Salze bilden lassen.

\section{Experimenteller Teil.}

Wenn man eine wässrige Lösung von Jodkalium mit einer Lösung von Ferricyankalium, welche im Liter $65,8 \mathrm{~g} \mathrm{Fe}(\mathrm{CN})_{8} \mathrm{~K}_{8}$ und $15 \mathrm{~g}$ $\mathrm{KOH}$ enthält, zusammenbringt, beobachtet man kaum irgend welche Veränderung.

Setzt man dagegen zu einer Jodkaliumlösung eine wässrige Lösung von Kaliumpermanganat, so wird letztere unter Abscheidung brauner Flocken von Manganoxyden sofort reduziert. Hier findet also eine sofortige Oxydation unter Bildung von jodsaurem Kalium statt, während bei Anwendung des in alkalischer Lösung fast ebenso kräftig wirkenden Kaliumferricyanids die Oxydationswirkung unterbleibt.

Ich kam daher auf den Gedanken, es möchte das Misslingen der Oxydation des Jodkaliums mittelst alkalischer Lösung von rotem Blutlaugensalz darauf zurückzuführen sein, dass zwischen jodsaurem Salz und dem bei der Oxydationswirkung des Ferricyankaliums stets gebildeten gelbem Blutlaugensalz eine umgekehrte Reaktion stattfindet. Vor allem aber sah ich das Hindernis der beabsichtigten Reaktion in der zu grossen Löslichkeit des zu erwartenden jodsauren Kaliums.

Es findet in der That bereits in neutraler Lösung zwischen Kaliumjodat und Ferrocyankalium, wie ich fand, eine gewisse Einwirkung statt, indem sich des letzteren fast farblose Lösung beim Vermischen mit einer Lösung von jodsaurem Kalium goldgelb färbte. Als ich dann verdünnte Schwefelsăure zusetzte, wurde die Mischung orangerot und trat beim Schütteln mit Chloroform an letzteres Jod ab.

Es war also ein gewisser Betrag an jodsaurem Kalium durch Abgabe von Sauerstoff an das Ferrocyankalium unter Bildung von Ferricyansalz (goldgelbe Färbung) zu Jodkalium reduziert worden, sodass beim nachherigen Zusatz von Schwefelsäure der übrig gebliebene Teil 
des Jodats auf das gebildete Jodkalium einwirken konnte, was bekanntlich unter Freiwerden von Jod geschieht.

Den hier erwăhnten Schwierigkeiten glaubte ich am besten zu entgehen, wenn ich eine Base zersetzte, mit der die zu erwartende Jodsäure nur schwer lösliche Verbindungen einzugehen vermag. Als ich daher zu einer Mischung von Ferricyankalium, Kalilauge und Jodkalium etwas Baryumnitrat fügte, erhielt ich nach 24 stündigem Stehen der erwärmten Flüssigkeit einen Ansatz hübscher goldgelber Krystalle von Baryumkaliumferrocyanür und zwischen ihnen verteilt ein weissliches krystallinisches Pulver, welches sich als Baryumjodat erwies. Es gab nach der mechanischen Trennung durch Auslesen der Krystalle und Waschen mit Wasser beim Uebergiessen mit Salzsäure Chlor, zeigte ferner die Barytreaktion und endlich bei Zusatz von Jodkalium und Versetzen mit einer Säure Abscheidung von Jod. Infolge dieser Beobachtung war der Weg zum gesteckten Ziele vorgezeichnet und galt es jetzt nur noch die beste Methode zur Bildung und Fällung des Jodats zu ermitteln. -

Es wurden die einzelnen Bestandteile zunächst nach der Gleichung $12 \mathrm{Fe}(\mathrm{CN})_{6} \mathrm{~K}_{8}+2 \mathrm{KJ}+7 \mathrm{Ba}(\mathrm{OH})_{2} 8$ aq. $=$

$$
\mathrm{Ba}\left(\mathrm{JO}_{8}\right)_{2}+2 \mathrm{KOH}+6\left[\left\{\mathrm{Fe}(\mathrm{CN})_{6} \mathrm{~K}_{3}\right\}_{2} \mathrm{Ba}\right]+55 \text { aq. }
$$

abgewogen und soviel Wasser, welches etwa das Dreifache der angewandten Menge Ferricyankalium betrug, zugemischt, dass die Bestandteile so ziemlich gelöst waren. Nach kurzem Schütteln im geschlossenen Zylinder zeigte sich die Bildung eines gelblich weissen, krystallinischen Niederschlags, welcher bei längerem Stehen noch erheblich zunahm. Das Pulver bestand grösstenteils aus Ferrocyankaliunıbaryum und enthielt jodsaures Baryum eingeschlossen. Die Trennung beider Körper ist indessen wegen der Schwerlöslichkeit des Barytdoppelsalzes eine missliche Operation und mit Verlusten an dem Jodat verbunden; auch kann letzteres bei der wiederholten Behandlung mit kochendem Wasser kaum farblos erhalten werden. Es besitzt meist wegen der intolge Erwärmung des alkalischen Gemisches eingetretenen Abscheidung von Eisenoxydhydrat ${ }^{1}$ ) eine gelblich-bräunliche Färbung, die beim Behandeln mit verdünnter Essigsăure einem Blaugrün (von Berlinerblau herrühend) Platz macht. Das von dem krystallinischen Pulvergemisch abgelaufene Filtrat gab beim Vermischen mit verdünnter Schwefelsäure weissliche Trübung von Baryumsulfat und beim Schüttelı mit Chloroform an letzteres Jod ab, welches, mit Natriumthiosulfatlösung bestimmt, noch circa $1 / 10$ der in Gestalt des Jodkaliums angewandten Menge betrug. Es war somit noch unangegriffenes Jod-

1) Vgl. Archiv der Pharmazie. Bd. 234, S. 335. 
kalium neben Ferricyankalium in der Lösung vorhanden, die Reaktion ist daher keine vollständige gewesen.

Als Hauptïbelstand erschien mir bei diesem Versuch die Bildung des schwer löslichen Barytdoppelsalzes, dessen Enstehung ich aber verhindern konnte, wenn ich den in obiger Gleichung erforderlichen hohen Betrag an Baryumhydrat auf das zur Bildung des Jodats notwendige Mafs reduzierte. Demgemăss wog ich jetzt die reagierenden Stoffe im Sinne folgender Gleichung $a b$ :

$$
\begin{gathered}
12 \mathrm{Fe}(\mathrm{CN})_{6} \mathrm{~K}_{8}+2 \mathrm{KJ}+12 \mathrm{KOH}+\mathrm{Ba}(\mathrm{OH})_{2} 8 \mathrm{aq.}= \\
\mathrm{Ba}\left(\mathrm{JO}_{8}\right)_{2}+2 \mathrm{KOH}+12 \mathrm{Fe}(\mathrm{CN})_{8} \mathrm{~K}_{4}+14 \mathrm{aq} .
\end{gathered}
$$

Und zwar kamen zur Verwendung 3,95 g Ferricyankalium, $0,33 \mathrm{~g}$ Jodkalium, 0,35 g krystallisiertes Baryumhydrat, 12,0 ccm Normal-Kalilauge und rund $10 \mathrm{ccm}$ Wasser, sodass das Gesamtvolumen $23 \mathrm{ccm}$ betrug.

Hier entstand nun kein Niederschlag mehr von einer schwerlöslichen Ferrocyandoppelverbindung, wohl aber bildete sich ein feinpulveriger krystallinischer von jodsaurem Baryum, welcher auf einem Filter gesammelt, gewaschen und als solcher identifiziert wurde.

Das Filtrat indessen zeigte noch goldgelbe Farbe und enthielt keinen Baryt mehr, obwohl es mit verdünnter Schwetelsäure noch ziemlich starke Jodfällung gab. Es schien daher ein Teil des Baryts in Form anderer Verbindungen, vielleicht von $\mathrm{BaCO}_{\mathrm{s}}$, mit niedergerissen zu sein, obwohl mit aller Vorsicht operiert wurde und nur ein chemisch reines, frisch umkrystallisiertes und uber Aetzkalk aufbewahrtes Baryumhydrat zur Verwendung gelangte.

Ich änderte daher das zuletzt erwähnte Mengenverhältnis dahin $\mathrm{ab}$, dass ich an Stelle von $0,35 \mathrm{~g} \mathrm{Ba}(\mathrm{OH})_{2} 8$ aq. jetzt $0,6 \mathrm{~g}$, also einen kleinen Ueberschuss nahm und dafür auch den Betrag an Aetzkali um 0,5 g vermehrte, um durch vermehrten Alkaligehalt die Bildung einer Ferrocyankaliumbaryum-Verbindung zu verhüten. Durch Probeversuche hatte ich nămlich gefunden, dass in einer überschüssiges Kaliumhydrat enthaltenden Flüssigkeit die Neigung zur Bildung der schwerlöslichen Doppelverbindung erheblich vermindert ist, dass Ferrocyankaliumbaryum geradezu durch Kalilauge in gewissem Betrage zersetzt wird.

In der That gelang es mir nun, eine ziemlich gute Reaktion zwischen den einzelnen Komponenten zu erreichen; ich erhielt 0,5 $\mathrm{g}$ nur wenig verunreinigtes Jodat, während die Gleichung theoretisch $0,485 \mathrm{~g}$ erwarten lässt.

Freilich enthielt auch hier noch das erste Filtrat, kenntlich an der goldgelben Farbe, etwas Ferricyansalz, und entstand noch mit $\mathrm{H}_{2} \mathrm{SO}_{4}$ neben $\mathrm{BaSO}_{4}$ etwas freies Jod; doch im Verhältnis zu den früheren Ergebnissen nur in verschwindender Menge. 
Uebrigens sei bemerkt, dass diese Jodabscheidung beim Hinzufüger von verdünnter Schwefelsäure mehrere Ursachen haben kann.

Es ist nämlich möglich, dass das noch in Lösung befindliche Baryumjodat mit einem Rest des ursprünglichen Jodkalinms in Wechselwirkung tritt, es ist aber auch ebenso wahrscheinlich, dass das noch sichtbar vorhandene Ferricyansalz diese Wirkung hervorbringt, oder dass erst im Augenblicke des Ansänerns ein Jodid resp. Jodwasserstoff gebildet wird, welcher dann mit Jodsäure oder Ferricyanwasserstoff reagieren kann.

Es lässt sich weiter denken, dass das Baryumjodat, soweit es nicht gefällt wurde, einen Teil des Ferrocyanwasserstoffes nach dem Hinzafügen von Säure zu oxydieren vermag. Hierdurch muss notwendig $\mathrm{BaJ}_{2}$ und aus ihm $\mathrm{HJ}$ entstehen, und letzterer dann zur Abscheidung von Jod Veranlassung geben.

Wie dem auch sei, auf jeden Fall lässt sich, soweit die Löslichkeit des Baryumjodats unter den obwaltenden Umständen (Ueberschuss von Kalilauge, Gegenwart von Ferrocyankalium) in Betracht kommt, eine völlige Abscheidung allen Jods als Jodat nicht erwarten. Die Ausbeute, welche ich erhielt, kann demnach als eine verhältnismässig befriedigende angesehen werden.

In einem weiteren Versuch, bei welchem ich das Dreifache der in dem vorherigen Versuch benutzten Stoffe verwendete, erhielt ich $1,38 \mathrm{~g}$ schneeweisses Baryumjodat, whhrend die Theorie 1,455 verlangt.

Dasselbe enthielt indessen noch ca. $20 \% \mathrm{BaCO}_{3}$, welches sich trotz aller Cautelen gebildet hatte, und 3,15\% Feuchtigkeit.

I. $0,1 \mathrm{~g}$ des luftrocknen Produkts verbrauchten $19,2 \operatorname{cem} 1 / 10 \mathrm{n}$. $\mathrm{Na}_{2} \mathrm{~S}_{8} \mathrm{O}_{8}$-Lösung.

II. $0,303 \mathrm{~g}$ des lufttrocknen Produkts verloren beim Trocknen bis $1600^{\circ}$ C. $0,0095 \mathrm{H}_{8} \mathrm{O}$.

III. $0,303 \mathrm{~g}$ des lufttrocknen. Produkts verbrauchten zur Sättigung $5,00 \mathrm{ccm} \mathrm{1/10} \mathrm{n.} \mathrm{HNO}_{8}$, entsprechend $0,049 \mathrm{Ba} \mathrm{CO}_{8}$.

Zusammensetzung daher gemäss: I. $\mathrm{Ba}\left(\mathrm{JO}_{8}\right)_{\mathbf{2}}=77,6 \%$

$$
\begin{aligned}
\text { II. } \mathrm{H}_{2} \mathrm{O}=3,15 \% \\
\text { III. } \mathrm{BaCO}_{3}=16,10 \% \text {. }
\end{aligned}
$$

Summa $96,85 \%$.

Um die Ausbeute noch zu vermehren, kann man für die Darstellnng des Korpers selbstverständlich auch direkt vom Jod ausgehen, wodurch der beim Lösen in Kalilauge gemăss nachstehender Gleichung:

$$
6 \mathrm{~J}+6 \mathrm{KOH}=5 \mathrm{KJ}+\mathrm{KJO}_{3}+3 \mathrm{H}_{2} \mathrm{O}
$$

verfïgbare Sauerstoff der Reaktion zu gute kommt. 
Es wurden daher die Komponenten auf Grund folgender Gleichung zusammengewogen:

$$
\begin{gathered}
2 \mathrm{~J}+10 \mathrm{Fe}(\mathrm{CN})_{9} \mathrm{~K}_{8}+10 \mathrm{KOH}+\mathrm{Ba}(\mathrm{OH})_{2} 8 \mathrm{aq} . \\
\mathrm{Ba}\left(\mathrm{JO}_{8}\right)_{2}+10 \mathrm{Fe}(\mathrm{CN})_{8} \mathrm{~K}_{4}+14 \mathrm{aq} .,
\end{gathered}
$$

wobei $1,27 \mathrm{~g}$ Jod zur Verarbeitung kam. Dasselbe wurde zunächst in der Kalilauge gelöst, darauf das fein zerriebene kohlensäurefreie $\mathrm{Ba}(\mathrm{OH})_{2}$ zugegeben, alsdann die übrigen Bestandteile. Es wurde hier in recht konzentrierter Mischung gearbeitet, sodass dieselbe nur ca. $70 \mathrm{ccm}$ betrug und das Ferricyansalz sich nicht völlig löste. Trotzdem war nach 48 stündigem Stehen jeder rote Krystall verschwunden und zeigten sich nur Ferrocyankalium-Krystalle neben dem weissen $\mathrm{Ba}\left(\mathrm{JO}_{3}\right)_{2}$. Nach dem Auswaschen und Trocknen gewann ich 2,286 g durch $\mathrm{BaCO}_{3}$ verunreinigtes Jodat.

Auch etwas Eisenhydroxyd zeigt sich häufig als Verunreinigung des auf vorstehende Weise gebildeten Baryumjodats, namentlich wenn man käufliches Ferricyansalz verwendet oder wenn man das alkalische Gemisch dem Licht aussetzt.

Man kann diese Verunreinigung oder event. auch (bei schlechtem Auswaschen) noch vorhandene Ferrocyanverbindungen bez. das sich beim Uebergiessen mit verdünnten Säuren bemerkbar machende Berlinerblau dadurch beseitigen, dass man das Produkt abwechselnd mit verdünnter Kalilauge und Salpetersäure behandelt und schliesslich gut wäscht. Ich erhielt auf diese Weise schliesslich ein rein weisses Produkt, freilich mit einigem Verlust an Substanz.

$0,178 \mathrm{~g}$ des gereinigten und bei $110^{\circ} \mathrm{C}$. getrockneten Produkts erforderten bei der Titrierung zur Bindung des mit $\mathrm{HCl}$ aus K.J ausgeschiedenen Jods $42,75 \mathrm{ccm}{ }^{1} / 10 \mathrm{Na}_{2} \mathrm{~S}_{2} \mathrm{O}_{3}$, woraus sich $0,173 \mathrm{~g} \mathrm{Ba}\left(\mathrm{JO}_{3}\right)_{2}$ berechnen $=97,2 \%$.

Vorstehende Arbeit wurde, wie schon bemerkt, nicht in der Absicht unternommen, um eine neue Methode zur Gewinnung von Jodaten bez. Jodsäure auszuarbeiten und für den Gebrauch in der Praxis zu empfehlen. Dazu ist bei dem Vorhandensein anderer guter oder besserer Verfahren keine Veranlassung gewesen.

Vielmehr lag es mir daran, festzustellen, ob auch durch rotes Blutlaugensalz in alkalischer Lösung Jodate gebildet werden können. Dieser Nachweis ist nun durch die Resultate obiger Versuche erbracht. 
Berücksichtigt man die von mir im Archiv der Pharmazie Bd. 234 S. 347 aufgestellte Theorie der Oxydationswirkung alkalischer Ferricyansalzlösungen, so wird man schliessen müssen, dass das infolge hydrolytischer Dissociation in obigen Kombinationen abgespaltene und temporär vorhandene Eisenhydroxyd ein so kräftiges Oxydationsmittel ist, dass es unter den erwähnten Verhältnissen selbst Jod in Jodsäure zu verwandeln vermag, was sonst nur durch verhältnismässig starke Agentien $\left(\mathrm{KMnO}_{4}, \mathrm{KClO}_{8}, \mathrm{HNO}_{3}\right)$ möglich ist.

\section{Mitteilungen aus der pharmazeutischen Abteilung des Eidgenössischen Polytechnikums in Zürich.}

\section{Weitere Beiträge zur Kenntnis der Cubeben. \\ Von C. Hartwich.}

(Eingegangen den 15. III. 1898.)

Im Jahre 1896 hat mein damaliger Assistent, Herr Dr. K. Peine man n, in dieser Zeitschrift (pag. 204) „Beiträge zur pharmakognostischen und chemischen Kenntnis der Cubeben etc," veröffentlicht. Sehr bald nach Publikation dieser Arbeit ging mir eine sehr reichhaltige Kollektion von Cubeben und von Früchten, die zu ihrer Verfälschung und zu ihrem Ersatz dienen, seitens des Kolonial-Museums in Haarlem zu. Diese Sendung wurde noch vervollständigt durch eine Anzahl von Berichten, betreffend Verfalschung der Cubeben in Java, die dem genannten Museum seitens dortiger Behörden erstattet waren. Ferner kam dazu eine Sendung von falschen Cubeben aus dem Museum der Pharmaceutical society in London, die ich Herrn E. M. Holmes verdanke.

Ich benutze zunächst die Gelegenheit, Herrn E. H. van Eeden, dem Direktor, Herrn Dr. M. Gresh off, dem Subdirektor des KolonialMuseums, und Herrn E. M. Holmes noch einmal an dieser Stelle meinen Dank auszusprechen.

Die Durcharbeitung dieser Kollektionen hat soviel des Neuen und Interessanten ergeben, dass ich die Resultate den Fachgenossen im Anschluss an die Arbeit des Herrn Dr. Peinemann hiermit vorlege. Ich gebe zunächst einige Bemerkungen, die sich auf die echte Cubebe von Piper Cubeba L. beziehen. 